\title{
THE X-RAY SPECTRA OF CYGNUS XR-1 AND THE CRAB NEBULA
}

\author{
Laurence E. Peterson \\ (University of California, San Diego, La Jolla, Calif., U.S.A.)
}

In this paper we wish to present briefly the latest results which have been obtained on the hard X-ray spectra of two strong sources in the Northern skies. These observations, which have been discussed in detail previously (Peterson et al., 1967), were made from balloons launched at Palestine, Texas, to $3 \mathrm{gm} / \mathrm{cm}^{2}$ atmospheric depth during September 1966. The Crab Nebula and the Cygnus XR-1 were observed to have a differential number power law spectra with an index of about -2 over the $20-200 \mathrm{keV}$ range. Both sources have the same intensity within about $10 \%$. The Crab Nebula has been observed on two occasions, one year apart, and showed no change in intensity over this range at about a $5 \%$ significance level.

These observations were made using a thin scintillation detector shielded with an active anti-coincidence collimator. The detectors are mounted on an alt-azimuth gimbal in the balloon gondola and referenced within about a degree to the local vertical and magnetic field. During the observation the detectors are fixed in azimuth North or South at the elevation of the object. In this manner a source makes a meridian transit through the aperture of the detector. Data for the background subtraction is obtained before and after the transit. Good events from the central detector are telemetered from a 128-channel pulse-height analyzer in a digital format; additional rate monitoring and housekeeping data are also telemetered.

The observation of the source Cygnus XR-1 was made on September 13, 1966. The detector used in this observation had an effective area of $50 \mathrm{~cm}^{2}$ and angular response, full width at one-half maximum (FWHM), of $8 \cdot 4^{\circ}$. The resolution of this detector was about $60 \%$ at $123 \mathrm{keV}$. Our results are shown in Figure 1 after correction for atmospheric absorption, with a power law fitted to the data over the range $20-200 \mathrm{keV}$. Data points obtained from rocket observations by Grader et al. (1966) and balloon observations by the group at M.I.T. (Clark et al., 1967), and the Dutch group (Bleeker et al., 1967) are also shown. Our points tend to fall slightly below the aggregate of the other measurements, though not beyond the statistical significance quoted by these workers. The two points at the lowest energy in our data have large systematic effects because of the poor detector resolution and large atmospheric correction which are not accounted for by the statistical errors shown. These results indicate clearly that the X-ray spectrum of Cygnus XR-1 has a power law characteristic like the Crab Nebula.

Perek (ed.), Highlights of Astronomy, 202-205. (C) I.A.U. 
Our observations on the Crab Nebula are shown in Figure 2. The new observation reported here was obtained on September 20, 1966 with the same detector used in our initial observation of the Crab Nebula one year previously. The detector area was $10 \mathrm{~cm}^{2}$, its aperture $24^{\circ} \mathrm{FWHM}$ and resolution was about $40 \%$ at $30 \mathrm{keV}$. The

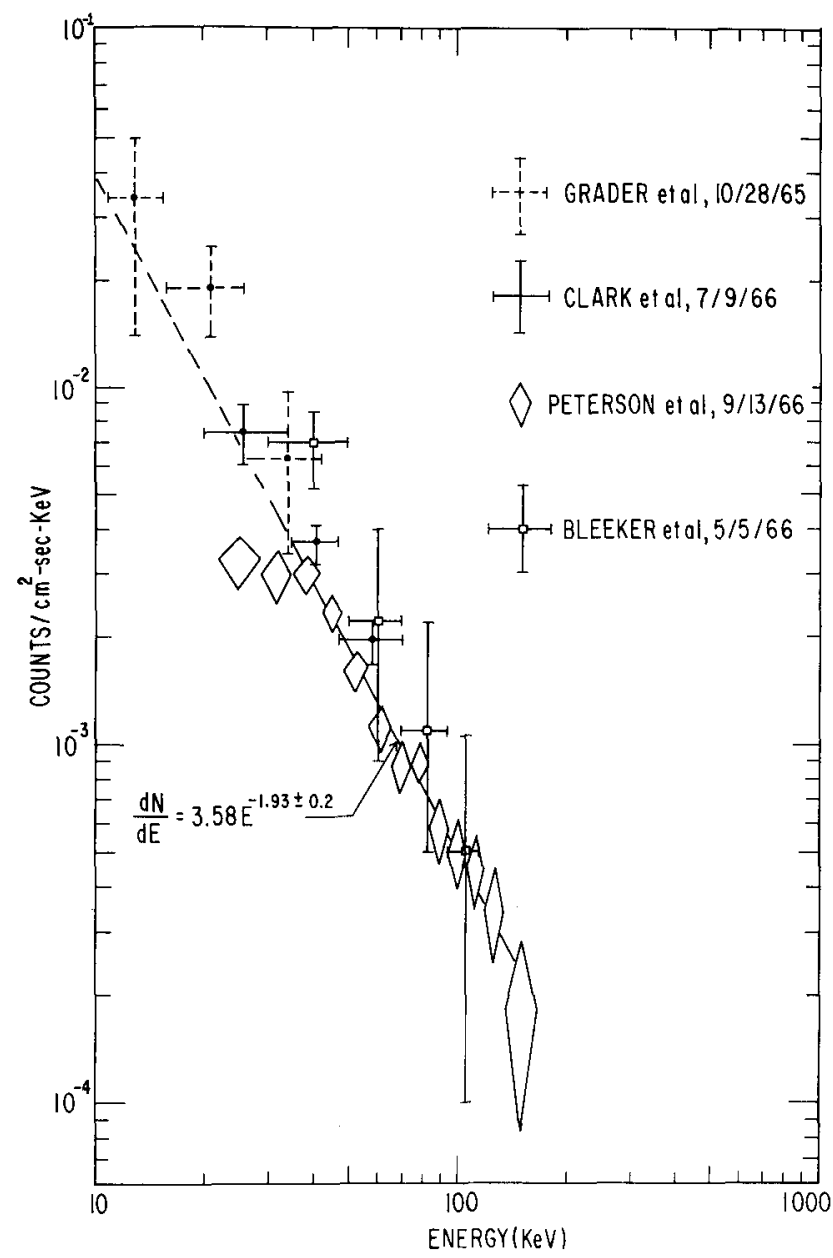

FIG. 1. This and previous measurements of the spectrum of the source Cygnus XR-1. Our data are best described as a power law whose differential energy index is $\alpha=0.9$ over the 30 to $140 \mathrm{keV}$ range.

results obtained during the most recent observation is shown by the diamonds in Figure 2. Also shown is a solid line which is a least squares fit to the data obtained on September 23, 1965. Clearly, there has been no obvious change over the 1-year period; the integrated flux of the Crab Nebula over the $20-100 \mathrm{keV}$ range, $0 \cdot 205$ 
counts $/ \mathrm{cm}^{2}$-sec, being constant to $\pm 5 \%$ at $3 \sigma$ confidence level. Since our observations were made with the same apparatus, systematic errors are minimized. Also shown are the data points obtained by George Clark in June 1964.

It is remarkable that the Crab Nebula and Cygnus XR-1 have a hard X-ray spectrum identical in magnitude and slope to within about $10 \%$. This suggests a comparable distance and a similar X-ray producing mechanism. This is different from Scorpius XR-1 whose exponential spectrum suggested emission from a hot, thin gas at $50 \times 10^{6} \mathrm{~K}$

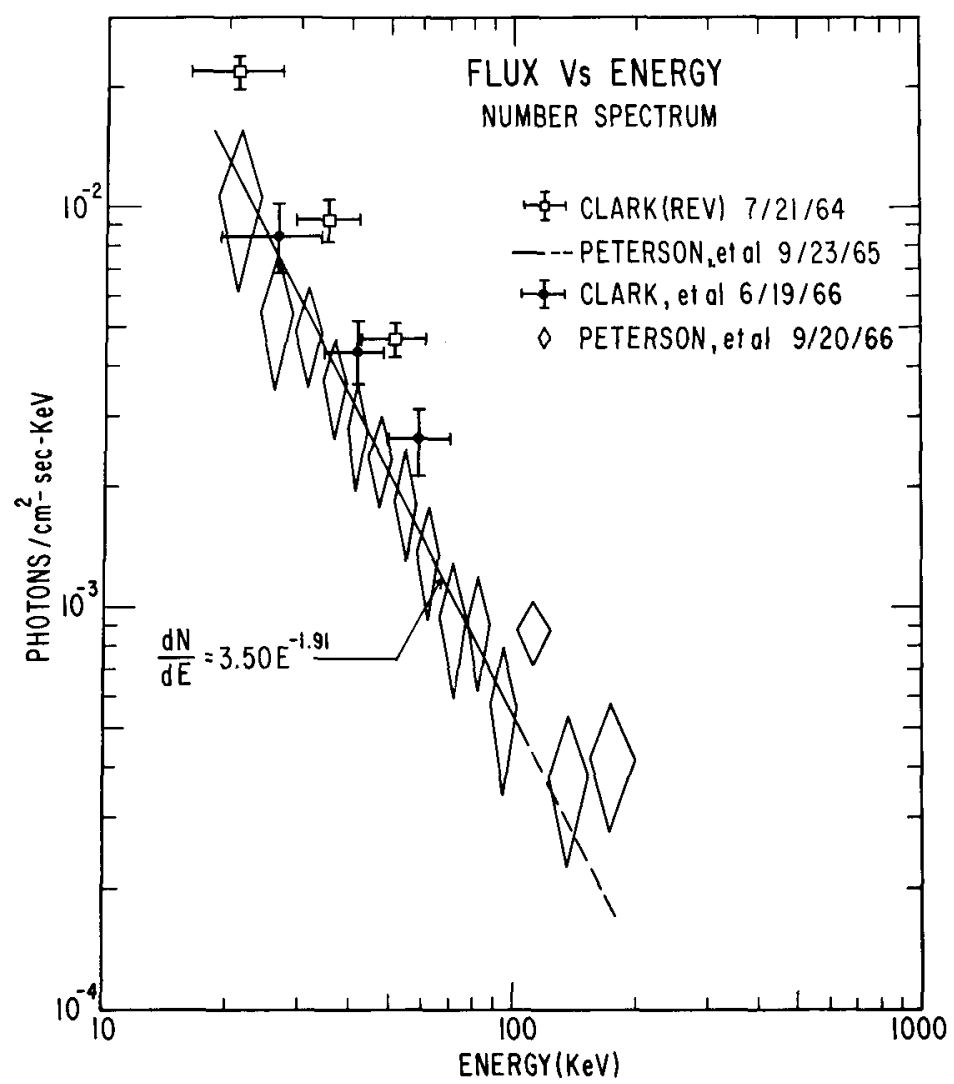

FIG. 2. Measurements of the Crab Nebula X-ray spectrum. Our results indicate the flux was constant to within $\pm 5 \%$ over a l-year interval.

(Peterson and Jacobson, 1966). Radio emission from the Crab Nebula is very different from that of Cygnus XR-1, which is presently unidentified with any obvious radio source. Comparisons at optical wavelengths cannot be made because Cygnus XR-1 is located in an obscured region of the galaxy (Giacconi et al., 1967). 


\section{Acknowledgments}

The author acknowledges the contribution of his associates, A.S. Jacobson, R.M. Pelling, and D.A. Schwartz in this work. The research was supported by the National Aeronautics and Space Administration under Grant NsG-318 and Contract NAS 5-3177.

\section{References}

Bleeker, J. A. M., Burger, J.J., Deerenberg, A.J. M., Scheepmaker, A., Swanenburg, B. N., Tanaka, Y. (1967) Astrophys. J., 147, 391.

Clark, G.W., Lewin, W.H.G., Smith, W.B. (1967) M.I.T. Lab. for Nuc. Sci. Preprint.

Giacconi, R., Gorenstein, P., Gursky, H., Waters, J. R. (1967) Astrophys. J., 148, L119.

Grader, R.J., Hill, R.W., Seward, F.D., Toor, A. (1966) Science, 152, 1499.

Peterson, L.E., Jacobson, A.S. (1966) Astrophys. J., 145, 962 965.

Peterson, L. E., Jacobson, A.S., Pelling, R. M., Schwartz, D. A. (1967) Canadian J. Phys. (in press). 